MATHEMATICS OF COMPUTATION

Volume 81, Number 278, April 2012, Pages 1025-1051

S $0025-5718(2011) 02535-4$

Article electronically published on August 25, 2011

\title{
EXPLICIT UPPER BOUNDS FOR THE REMAINDER TERM IN THE DIVISOR PROBLEM
}

\author{
D. BERKANE, O. BORDELLÈS, AND O. RAMARÉ \\ To the memory of John Selfridge
}

\begin{abstract}
We first report on computations made using the GP/PARI package that show that the error term $\Delta(x)$ in the divisor problem is $=\mathscr{M}(x, 4)+$ $O^{*}\left(0.35 x^{1 / 4} \log x\right)$ when $x$ ranges $\left[1081080,10^{10}\right]$, where $\mathscr{M}(x, 4)$ is a smooth approximation. The remaining part (and in fact most) of the paper is devoted to showing that $|\Delta(x)| \leq 0.397 x^{1 / 2}$ when $x \geq 5560$ and that $|\Delta(x)| \leq$ $0.764 x^{1 / 3} \log x$ when $x \geq 9995$. Several other bounds are also proposed. We use this results to get an improved upper bound for the class number of a quadractic imaginary field and to get a better remainder term for averages of multiplicative functions that are close to the divisor function. We finally formulate a positivity conjecture concerning $\Delta(x)$.
\end{abstract}

\section{INTRODUCTION}

The object of this paper is to study for an explicit viewpoint the remainder term of the summatory function of the $\tau$-function, where $\tau(n)$ denotes the number of (positive) divisors of $n$, i.e., to study

$$
\Delta(x)=\sum_{n \leq x} \tau(n)-x(\log x+2 \gamma-1) .
$$

This function has been extensively studied, and the reader will find a good survey in [7. It is known in particular that

$$
\Delta(x) \ll_{\varepsilon} x^{131 / 416+\varepsilon}
$$

for any $\varepsilon>0$. We want to get fully explicit bounds of this shape here, and the best exponent we reach is $1 / 3$ (see Theorem 1.2 below). Note that $131 / 416=0.314 \cdots$ is not so much smaller than $1 / 3=0.333 \cdots$. Note further that Theorem 1.1 below gives an upper bound with a worse exponent, but which is better on a large range. The divisor function has been studied from this viewpoint in several papers, and we quote here [12, [19, 4] and [15].

Here are our main results:

Theorem 1.1. When $x \geq 1$, we have $|\Delta(x)| \leq 0.961 x^{1 / 2}$.

When $x \geq 1981$, we have $|\Delta(x)| \leq 0.482 x^{1 / 2}$.

When $x \geq 5560$, we have $|\Delta(x)| \leq 0.397 x^{1 / 2}$.

Received by the editor January 3, 2011 and, in revised form, February 16, 2011.

2010 Mathematics Subject Classification. Primary 11N56; Secondary 11N37.

(C)2011 American Mathematical Society

Reverts to public domain 28 years from publication 
These bounds are sharp, since $|\Delta(x)|>0.5 x^{1 / 2}$ when $x=1980$ while $|\Delta(x)|>$ $0.4 x^{1 / 2}$ when $x=5559$.

Theorem 1.2. When $x \geq 9995$, we have $|\Delta(x)| \leq 0.764 x^{1 / 3} \log x$.

This bound is also sharp since $|\Delta(x)|>0.80 x^{1 / 3} \log x$ when $x=9994$. This bound is of course asymptotically better than the one given by Theorem 1.1 but this latter one still prevails when $x \leq 59576122384$.

There are two usual paths to study $\Delta(x)$ that can be broadly described by either using a Voronoï-like formula as in [13, or using the fractional part-function, expanding it in a Fourier series and using exponential sums, and using, for instance, [1, Lemma 8.4] (see also [2] for similar material, as well as [6, section 8]). We use the first technique, but rely on an earlier paper of Voronoï where a very explicit result is proved.

We rely also on some rather extensive computations detailed in section [6 made with the help of the PARI/GP program (see [20]) and its auxiliary GP2C. One of the main problems with such extensive computations is always how to store them, since tables are difficult to use. We again use the Voronoï formula to get such a model and prove the following.

Theorem 1.3. For all $x \in\left[3,10^{10}\right]$ we have

$$
\Delta(x)=\mathscr{M}(x, 4)+O^{*}\left(0.9 x^{1 / 4} \log x\right)
$$

and for all $x \in\left[1081080,10^{10}\right]$, we have

$$
\Delta(x)=\mathscr{M}(x, 4)+O^{*}\left(0.35 x^{1 / 4} \log x\right) .
$$

Here

$$
\begin{aligned}
\mathscr{M}(x, 4)=\frac{x^{1 / 4}}{\pi \sqrt{2}}(\cos (4 \pi \sqrt{x} & \left.-\frac{\pi}{4}\right)+2^{1 / 4} \cos \left(4 \pi \sqrt{2 x}-\frac{\pi}{4}\right) \\
& \left.+\frac{2}{3^{3 / 4}} \cos \left(4 \pi \sqrt{3 x}-\frac{\pi}{4}\right)+\frac{3}{4^{3 / 4}} \cos \left(4 \pi \sqrt{4 x}-\frac{\pi}{4}\right)\right) .
\end{aligned}
$$

Section [6 contains more bounds of this shape. Note that the constant 0.35 is very good and fairly stable, since, for instance,

$$
\left|\Delta\left(x_{0}\right)-\mathscr{M}\left(x_{0}, 4\right)\right| \geq 0.289 x_{0}^{1 / 4} \log x_{0} \quad \text { when } x_{0}=9137256975
$$

A constant of 0.30 would require us to start at least at $2.7 \cdot 10^{9}$, which renders the preliminary computations difficult. It would be valuable to extend Theorem 1.3 to a larger range. 
We end this introduction by mentioning a curious conjecture upon which we stumbled:

Conjecture 1.4. For all $T \geq 1$, we have

$$
\int_{T}^{\infty} \frac{\Delta(u) d u}{u^{7 / 4}} \geq 0
$$

See section 8 for more background on this conjecture.

Notation. We use the Landau-like notation $f=O^{*}(g)$ to say that $|f| \leq g$. We use $\psi(x)=x-[x]-1 / 2$, where $[x]$ is the integer part of $x$. We shall also need the multiplicative function

$$
\tilde{\tau}(n, D)=\sum_{\substack{u v=n,(u, v, D)=1}} 1
$$

for some parameter $D$, where $(u, v, D)$ denotes the gcd of $u, v$ and $D$.

\section{TWO APPLiCATIONS}

An application to number fields. Let $K / \mathbb{Q}$ be a number field of degree $n$, class number $h_{K}$, signature $\left(r_{1}, r_{2}\right)$ and let $d_{K}$ be the absolute value of its discriminant. We set $b_{K}$ to be a real number such that each ideal class contains a nonzero ideal $A$ satisfying $\mathcal{N}(A) \leqslant b_{K} \sqrt{d_{K}}$, where $\mathcal{N}$ denotes the ideal-norm operator in $K$. It is well known that one can take for $b_{K}$ the Minkoswki bound $(4 / \pi)^{r_{2}} n ! n^{-n}$. If $K$ is an imaginary quadratic field, then the better bound $b_{K}=3^{-1 / 2}$, due to Gauss, can be used instead of the Minkowski constant.

It has been shown by the second author of [1] that the inequality

$$
h_{K} \leqslant 2^{2-n} b_{K} d_{K}^{1 / 2}\left(\log \left(b_{K}^{2} d_{K}\right)\right)^{n-1}
$$

holds for all number fields $K$ subject to the condition $d_{K} \geqslant 36 b_{K}^{-2}$. In the case of real quadratic fields, using Dirichlet's analytic class number formula and precise estimates for $L(1, \chi)$ (where $\chi$ is the primitive real Dirichlet character attached to $K)$ and the fundamental unit of $K$, Maohua Le [1] proved that

$$
h_{K} \leqslant \sqrt{d_{K}} / 2 .
$$

A simpler proof of this bound has been provided by the third author in [16. Using Theorem 1.3 we deduce the following slight improvement of (2.1) in the case of imaginary quadratic fields.

Corollary 2.1. Let $K=\mathbb{Q}(\sqrt{-d})$ be an imaginary quadratic field with $d>0$ squarefree and $d_{K}$ is the absolute value of its discriminant. If $d_{K} \geqslant 108$, then we have

$$
h_{K} \leqslant \sqrt{\frac{d_{K}}{12}} \log d_{K}
$$

Examples. In what follows, we set $\mathcal{B}_{K}=\left[\sqrt{d_{K} / 12} \log d_{K}\right]$, where $K$ is an imaginary quadratic subfield of the cyclotomic field $\mathbb{Q}\left(\zeta_{d}\right)$ where $\zeta_{d}$ is a primitive $d$-th root of unity. The computations have been made using PARI system. 


\begin{tabular}{|c|c|c|}
\hline$d$ & $h_{K}$ & $\mathcal{B}_{K}$ \\
\hline \hline 311 & 19 & 29 \\
\hline 1559 & 51 & 83 \\
\hline 149159 & 597 & 1328 \\
\hline 300119 & 781 & 1994 \\
\hline
\end{tabular}

An application to averages of multiplicative functions. [15, Lemma 3.2] proposes an automatic way of deriving an explicit bound for averages of multiplicative nonnegative functions that are close enough to a given model. The two models proposed are the constant function 1 and the divisor function. In this latter case, using this lemma requires an explicit bound for $\sum_{n \leq t} \tau(n) / n$ and the above paper relies on [19. Lemma 1] (this is also the second part of [15, Lemma 3.3]). We improve this lemma to the following.

Corollary 2.2. We have, for all $t>0$,

$$
\sum_{n \leq t} \frac{\tau(n)}{n}=\frac{1}{2} \log ^{2} t+2 \gamma \log t+\gamma^{2}-\gamma_{1}+O^{*}\left(1.16 / t^{1 / 3}\right)
$$

where $\gamma_{1}$ is the second Laurent-Stieljes constant, for instance, [10] and [3]. In particular, we have

$$
\gamma_{1}=-0.0728158454836767248605863758749013191377+O^{*}\left(10^{-40}\right) .
$$

\section{Borrowing From Dirichlet}

Let us first recall a result of Dirichlet.

Lemma 3.1 (Dirichlet). When $x \geq 1$ is a real number, we have

$$
\left|\Delta(x)+2 \sum_{n \leq \sqrt{x}} \psi(x / n)\right| \leq \frac{1}{2}
$$

The proof we present is somewhat more complete than that of [1, Lemma 8.1], since we express $\mathcal{R}(x)$ below fully in terms of $\psi_{2}$.

Proof. Set $\psi_{2}(t)=\frac{1}{2} \psi(t)^{2}$. We first notice that the function $x \mapsto \frac{1}{8}+\int_{1}^{x} \psi(t) d t$ is periodic of period 1 , and that, when $0 \leq y<1$,

$$
\int_{1}^{1+y} \psi(t) d t+\frac{1}{8}=\int_{0}^{y}\left(t-\frac{1}{2}\right) d t+\frac{1}{8}=\psi_{2}(y)=\psi_{2}(1+y)
$$


and thus $\psi_{2}$ is an antiderivative of $\psi$. By Dirichlet's Hyperbola Principle and Euler-MacLaurin's Summation Formula we get

$$
\begin{aligned}
\sum_{n \leqslant x} \tau(n)= & 2 \sum_{n \leqslant x^{1 / 2}}[x / n]-[\sqrt{x}]^{2} \\
= & 2 x \sum_{n \leqslant x^{1 / 2}} \frac{1}{n}-2 \sum_{n \leqslant x^{1 / 2}} \psi\left(\frac{x}{n}\right)+\frac{1}{2}-x+2 \sqrt{x} \psi(\sqrt{x})-\psi(\sqrt{x})^{2}-\frac{1}{4} \\
= & 2 x\left(\frac{\log x}{2}+\gamma-\frac{\psi(\sqrt{x})}{\sqrt{x}}-\frac{\psi_{2}(\sqrt{x})}{x}+2 \int_{\sqrt{x}}^{\infty} \frac{\psi_{2}(t)}{t^{3}} \mathrm{~d} t\right) \\
& -2 \sum_{n \leqslant x^{1 / 2}} \psi\left(\frac{x}{n}\right)+\frac{1}{4}-x+2 \sqrt{x} \psi(\sqrt{x})-\psi(\sqrt{x})^{2} \\
= & x(\log x+2 \gamma-1)-2 \sum_{n \leqslant x^{1 / 2}} \psi\left(\frac{x}{n}\right)+\mathcal{R}(x),
\end{aligned}
$$

where

$$
\mathcal{R}(x)=\frac{1}{4}-3 \psi_{2}(\sqrt{x})+4 x \int_{\sqrt{x}}^{\infty} \frac{\psi_{2}(t)}{t^{3}} \mathrm{~d} t .
$$

The inequality $0 \leqslant \psi_{2}(t) \leqslant 1 / 8$ implies that

$$
\left|\frac{1}{4}-3 \psi_{2}(\sqrt{x})\right| \leqslant \frac{1}{4}
$$

and

$$
4 x\left|\int_{\sqrt{x}}^{\infty} \frac{\psi_{2}(t)}{t^{3}} \mathrm{~d} t\right| \leqslant \frac{x}{2} \int_{\sqrt{x}}^{\infty} \frac{\mathrm{d} t}{t^{3}}=\frac{1}{4},
$$

which concludes the proof.

Corollary 3.2. When $x \geq 1$ is a real number, we have $|\Delta(x)| \leq \sqrt{x}+\frac{1}{2}$.

\section{Auxiliary Results}

Let us start with a generic formula, valid for any sequence $\left(\varphi_{n}\right)$. We define an abstract remainder term by

$$
\Delta_{\varphi}(t)=\sum_{n \leq t} \varphi_{n}-(a t \log t+b t)
$$

for some real numbers $a$ and $b$. The following formula holds for any complex number $s \neq 1$ :

$$
\begin{aligned}
\sum_{n \leq T} \frac{\varphi_{n}}{n^{s}}=\frac{a T^{1-s} \log T}{1-s}+\frac{b(1-s)-a s}{(1-s)^{2}} T^{1-s} & \\
& +\frac{s(a-b(1-s))}{(1-s)^{2}}+T^{-s} \Delta_{\varphi}(T)+s \int_{1}^{T} \Delta_{\varphi}(u) d u / u^{s+1} .
\end{aligned}
$$

This is most readily obtained by summation by parts. 
From $\tilde{\tau}(\cdot, D)$ to $\tau(\cdot)$. The gcd condition in $\tilde{\tau}(\cdot, D)$ is easily handled by using the Möbius function. Indeed, on using the following easily proved formula

$$
\mathbb{1}_{(u, v, D)=1}=\sum_{\substack{\delta|u, \delta| v, \delta \mid D}} \mu(\delta),
$$

we readily get, for $T>0$,

$$
\sum_{n \leq T} \frac{\tilde{\tau}(n, D)}{n^{s}}=\sum_{\delta \mid D} \mu(\delta) \sum_{\substack{\delta|u, \delta| v, u v \leq T}} \frac{1}{(u v)^{s}}=\sum_{\delta \mid D} \frac{\mu(\delta)}{\delta^{2 s}} \sum_{n \leq T / \delta^{2}} \frac{\tau(n)}{n^{s}} .
$$

On selecting $s=0$, this leads to the asymptotic formula

$$
\sum_{n \leq T} \tilde{\tau}(n, D)=A(D) T \log T+B(D) T+\Delta(T, D)
$$

where $A(D)$ and $B(D)$ are defined by

$$
A(D)=\sum_{\delta \mid D} \frac{\mu(\delta)}{\delta^{2}}, \quad B(D)=\sum_{\delta \mid D} \frac{\mu(\delta)}{\delta^{2}}(2 \gamma-1-2 \log \delta),
$$

while $\Delta(\cdot, D)$ is expressed in terms of $\Delta(\cdot)$ by

$$
\Delta(T, D)=\sum_{\delta \mid D} \mu(\delta) \Delta\left(T / \delta^{2}\right) .
$$

Some formulae with $\tilde{\tau}(n, D)$. We select $a=A(D), b=B(D), s=1 / 2$ and $s=3 / 4$ in formula (4.1) and quote explicitly:

$$
\begin{aligned}
\sum_{n \leq T} \frac{\tilde{\tau}(n, D)}{n^{1 / 2}}=2 A(D) T^{1 / 2} & \log T+2(B(D)-A(D)) T^{1 / 2} \\
& +2 A(D)-B(D)+\frac{\Delta(T, D)}{T^{1 / 2}}+\frac{1}{2} \int_{1}^{T} \frac{\Delta(u, D) d u}{u^{3 / 2}},
\end{aligned}
$$

which is the case $s=1 / 2$ from above. The case $s=3 / 4$ reads

$$
\begin{aligned}
\sum_{n \leq T} \frac{\tilde{\tau}(n, D)}{n^{3 / 4}}=4 A(D) & T^{1 / 4} \log T+4(B(D)-3 A(D)) T^{1 / 4} \\
& +12 A(D)-3 B(D)+\frac{\Delta(T, D)}{T^{3 / 4}}+\frac{3}{4} \int_{1}^{T} \frac{\Delta(u, D) d u}{u^{7 / 4}} .
\end{aligned}
$$

A generic integral. We note that, when $s \neq 1,2$,

$$
\int \frac{t(\log t+c)+d}{t^{s}} d t=\frac{\log t+(s-2)^{-1}+c}{(2-s) t^{s-2}}+\frac{d}{(1-s) t^{s-1}} .
$$

Proof. Take the derivative of the right-hand side and check that it is the integrand. 


\section{BORROWING FROM VORONOÏ}

The purely elementary method of Voronoï, which improves on the Dirichlet hyperbola formula by using triangles instead of rectangles beneath the hyperbola $m n=x$, yields the following result [21, pages 280, 281].

Lemma 5.1. When $x \geq 1, T \geq 1$ and $D \geq 1$ are real numbers, we have

$$
\begin{aligned}
|\Delta(x)| \leq \frac{19}{12} \sum_{n \leq T} \tilde{\tau}(n, D)+\left(\frac{\sqrt{x}}{4 T}+\frac{\sqrt{T}}{6}\right) & \sum_{n \leq T} \frac{\tilde{\tau}(n, D)}{\sqrt{n}} \\
& +\frac{3 x^{1 / 4}}{4} \sum_{n \leq T} \frac{\tilde{\tau}(n, D)}{n^{3 / 4}}+\frac{T}{6}+\sqrt{\frac{x}{T}}+\frac{7}{4}
\end{aligned}
$$

where $\tilde{\tau}$ is defined in (1.2).

Comparing with [22, page 209, Théorème] and 223, page 429, paragraph 49, théorème $\mathrm{I}]$, or with $[8$ or $[13$, we see that, in case $D=1$, one can asymptotically dispense with the first two sums at the cost of a $O_{\varepsilon}\left(x^{\varepsilon}\right)$ for any $\varepsilon>0$, and that the constant $3 / 4$ in front of the third sum can be reduced to $1 /(\pi \sqrt{2})$. The advantage of the above lemma relies on its range of validity. The parameter $D$ (or the fact that we can replace the $\tau$-function by the number of coprime divisors) is a distinct feature of the above bound. We shall select $D=6$, reducing the total bound by a factor about $\left(1-\frac{1}{4}\right)\left(1-\frac{1}{9}\right)=2 / 3$.

Proof. The paper [21] contains the required estimates, but the following notes may be helpful to the reader: equation (17) on page 280 contains the function $F$ which is generally defined in equation (1) at the very beginning of the paper; it is also given at the beginning of section 26, page 275. To read equation (17) the reader will need equation (10), page 279, which contains the definition of $R$. This definition comes in fact from (18), page 271 .

Voronoï continues by bounding $\tilde{\tau}$ by $\tau$ (see equation (19) and (20) of [21, pages 280, 281]). On using (4.6) and (4.7) and shortening $A(D)$ and $B(D)$ to $A$ and $B$, respectively, we reach

$$
\begin{array}{r}
|\Delta(x)| \leq \frac{T}{12}(23 A \log T+23 B-19 A+2)+3(x T)^{1 / 4}(A \log T+B-3 A) \\
+\sqrt{\frac{x}{T}}\left(A \frac{\log T}{2}+\frac{B-A}{2}+1\right)+\frac{36 A-9 B}{4} x^{1 / 4} \\
+\frac{2 A-B}{4} \frac{\sqrt{x}}{T}+\frac{2 A-B}{6} \sqrt{T}+G(D, x, T),
\end{array}
$$

with

$$
\begin{aligned}
G(D, x, T)=\frac{7}{4}+ & \left(7+\left(x T^{-3}\right)^{1 / 4}+\left(x T^{-3}\right)^{1 / 2}\right) \frac{\Delta(D, T)}{4} \\
+ & \left(\frac{\sqrt{x}}{8 T}+\frac{\sqrt{T}}{12}\right) \int_{1}^{T} \frac{\Delta(D, u) d u}{u^{3 / 2}}+\frac{9 x^{1 / 4}}{16} \int_{1}^{T} \frac{\Delta(D, u) d u}{u^{7 / 4}} .
\end{aligned}
$$

The introduction of the parameter $D$ in Lemma 5.1 will be numerically interesting. We will use only small $D$ 's, such as 1,2 or 6 . 


\section{NumericAlly COMPARING $\Delta$ WITH A MODEL}

We need to compute values of $\Delta(x)$ for fairly large $x$. The first idea is to compute it directly, take its absolute value, divide it by $\sqrt{x}$ and look for the point when it is less than a given bound, say 0.5. The drawback of this method is that one would have to redo all the computations with the bound 0.3 . To avoid that, one can store the value on short enough ranges, say every $5 \cdot 10^{7}$, but we would have to store these tables and they would be very bulky to use in computations. Musing on this idea, we readily discover that a better idea would be to compare $\Delta(x)$ with a model and bound the resulting error term. This is a very general idea, and one that we have already used in [17, Theorem 2]; the difficulty is always to guess a proper model. However, this issue is easily solved here, since a model is provided to us by the Voronoï formula. We define

$$
\mathscr{M}(x, M)=\frac{x^{1 / 4}}{\pi \sqrt{2}} \sum_{m \leq M} \frac{\tau(m)}{m^{3 / 4}} \cos \left(4 \pi \sqrt{m x}-\frac{\pi}{4}\right) .
$$

We look for numerical bounds for $|\Delta(x)-\mathscr{M}(x, M)| /\left[x^{1 / 4} \log x\right]$ for some small $M$. Note that $\mathscr{M}(x, M)$ is thus of size $x^{1 / 4}$ in that case. We have found that, when $M=1$ or $M=4$, the function $x^{1 / 4}$ is too small to evaluate $|\Delta(x)-\mathscr{M}(x, M)|$ while $x^{1 / 4} \log x$ seems just too large. The bounds obtained are, however, better when subtracting $\mathscr{M}(x, 1)$, and even better when subtracting $\mathscr{M}(x, 4)$.

The computations necessitate some care. For $x \in[N, N+1)$, we consider the function

$$
f(x)=\left[\sum_{n \leq N} \tau(n)-(x \log x+(2 \gamma-1) x)-\mathscr{M}(x, M)\right] /\left[x^{1 / 4} \log x\right] .
$$

We find that, with $S=\sum_{n \leq N} \tau(n)$,

$$
\begin{aligned}
x^{1 / 4} \log x f^{\prime}(x)=-\frac{S}{4 x} & -\frac{S}{x \log x}-\frac{3 \log x-6 \gamma+3}{4}+\frac{2 \gamma-1}{\log x} \\
& +\sum_{m \leq M} \frac{\sqrt{2} \tau(m)}{m^{1 / 4} x^{1 / 4}} \sin \left(4 \pi \sqrt{m x}-\frac{\pi}{4}\right) \\
& -\frac{1}{\sqrt{2} \pi \log x} \sum_{m \leq M} \frac{\tau(m)}{m^{3 / 4} x^{3 / 4}} \cos \left(4 \pi \sqrt{m x}-\frac{\pi}{4}\right) .
\end{aligned}
$$

Since $S \geq 2 x-1$, we are sure this derivative is nonpositive when

$$
\begin{aligned}
3 \log x-6 \gamma+5 \geq \frac{1}{x}+\frac{4}{x \log x}+\frac{8 \gamma+4}{\log x}+\sum_{m \leq M} \frac{4 \sqrt{2} \tau(m)}{m^{1 / 4} x^{1 / 4}} & \\
& +\frac{2 \sqrt{2}}{\pi \log x} \sum_{m \leq M} \frac{\tau(m)}{m^{3 / 4} x^{3 / 4}} .
\end{aligned}
$$

The difference between the left-hand side and the right-hand side is an increasing function, from which it follows immediately that there exists an integer $N_{0}(M)$ such that, when $N \geq N_{0}(M)$, the function $x \mapsto(\Delta(x)-\mathscr{M}(x, M)) /\left[x^{1 / 4} \log x\right]$ is nonincreasing in each interval $[N, N+1)$. The parameter $M$ being fixed, $N_{0}(M)$ is a fixed (and small) value, and, for instance, $N_{0}(1)=2$ and $N_{0}(4)=5$ (we find that, in the case $M=4, f^{\prime}(x)<0$ when $x \geq 11.062$, and is not an integer). Finding 
the maximum of $|\Delta(x)-\mathscr{M}(x, M)| /\left[x^{1 / 4} \log x\right]$ below this value can be automated, but it is more expedient, as well as less error-prone, to simply plot the function in each of the remaining unit intervals.

Numerical experiments show that $\mathscr{M}(x, 1)$ is already a good model! For small values, we find that

\begin{tabular}{|r|c||c|r|r|}
\hline \multicolumn{5}{|c|}{ Using the model $\mathscr{M}(x, 1)$} \\
\hline Beginning & End & Max $\leq$ & Where & Sum there \\
\hline 9 & 10001 & 0.689848 & 12 & 35 \\
10001 & 20001 & 0.442832 & $15120-$ & 147800 \\
20001 & 30001 & 0.440962 & 25200 & 259338 \\
30001 & 40001 & 0.405939 & $30240-$ & 316597 \\
40001 & 50001 & 0.400379 & 49140 & 538485 \\
50001 & 60001 & 0.406026 & 50400 & 553570 \\
60001 & 70001 & 0.379055 & $60480-$ & 675163 \\
70001 & 80001 & 0.379005 & $75600-$ & 860836 \\
80001 & 90001 & 0.382929 & $83160-$ & 954846 \\
90001 & 100001 & 0.410340 & 97020 & 1129117 \\
\hline
\end{tabular}

When the maximum have been attained at the end of the interval $[N, N+1)$, the program has attached a minus sign at the back of the data "Where". We have used the function MajoreDelta between 1 and $10^{10}$ below.

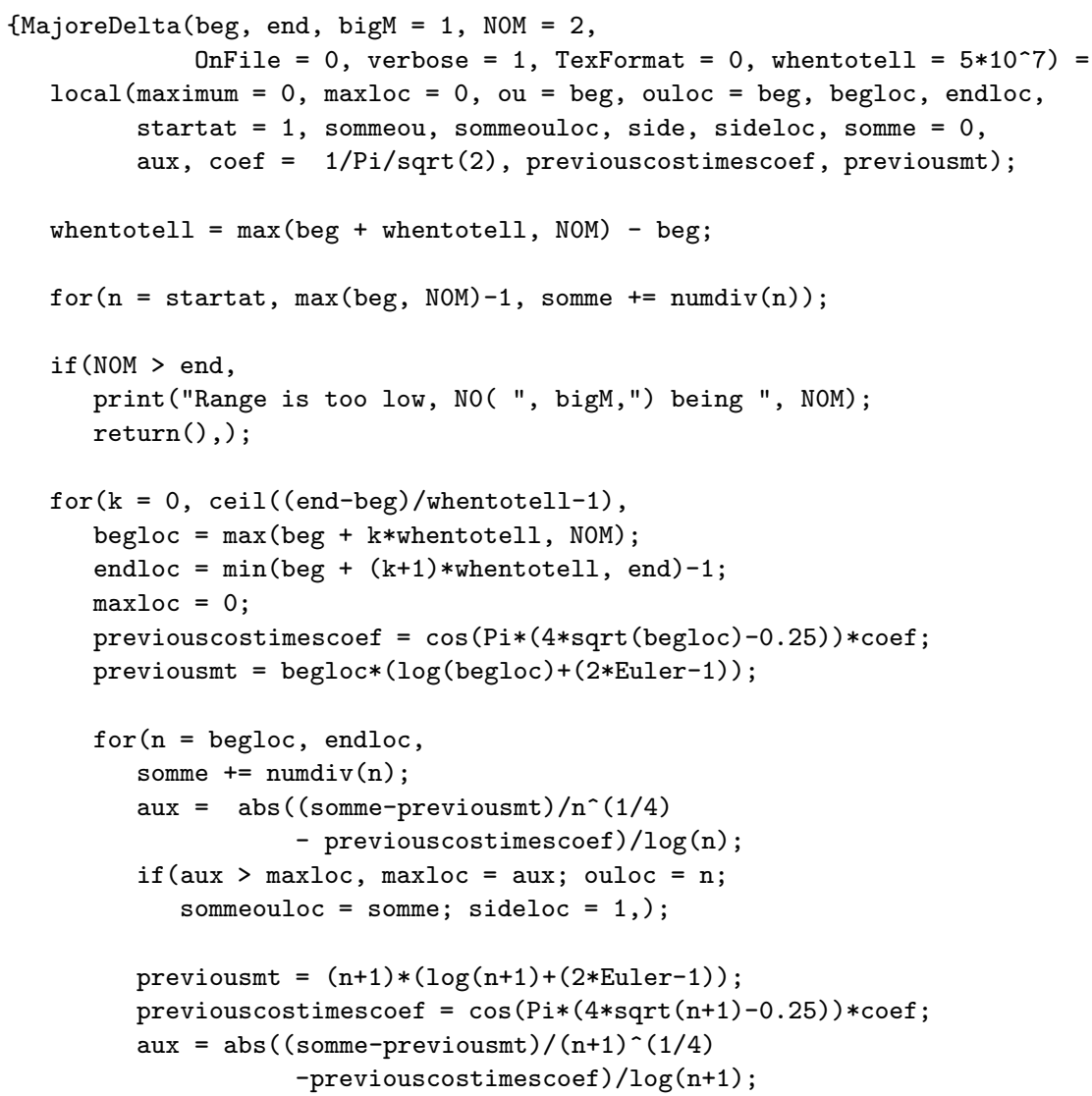




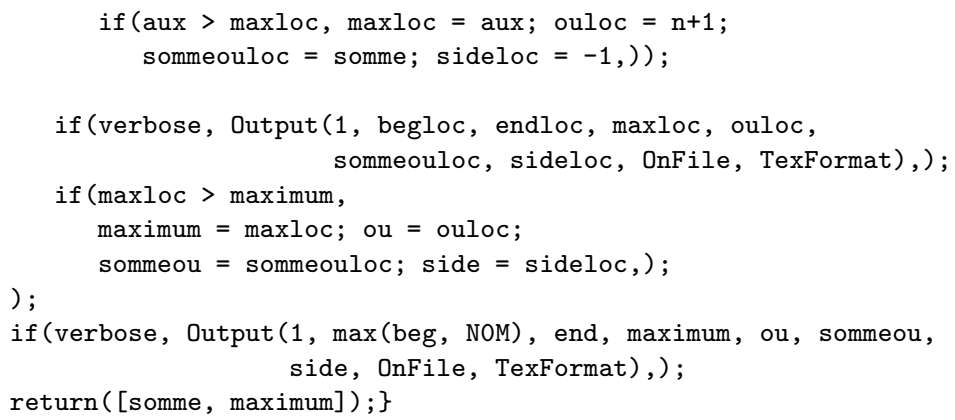

The code for the function Output will be easily guessed by the reader. It can also be obtained by sending an e-mail request to the third named author of this paper. We have converted this function into a C-program and have compiled it with GP2C via the command

$$
\text { gp2c -g ModeleDelta-special.gp > MajoreDelta-special.gp.c }
$$

This step speeds the computations by a large factor (about 10). We then started GP with the option $-\mathrm{p} 10000000000$ and installed the compiled functions as described in the GP2C manual.

Below is the table obtained, each entry requiring at the beginning nearly 40 minutes (on a desktop computer).

\begin{tabular}{|r|r||c|r|r|}
\hline \multicolumn{5}{|c|}{ Using the model $\mathscr{M}(x, 1)$} \\
\hline Beginning & End & Max $\leq$ & Where & Sum there \\
\hline 9 & 50000001 & 0.689848 & 12 & 35 \\
50000001 & 100000001 & 0.362373 & 82882820 & 1523997698 \\
100000001 & 150000001 & 0.335167 & 134603040 & 2540265823 \\
150000001 & 200000001 & 0.340907 & 165765640 & 3162894841 \\
200000001 & 250000001 & 0.302913 & 203898905 & 3932714293 \\
250000001 & 300000001 & 0.305402 & 274266920 & 5371256127 \\
300000001 & 350000001 & 0.324542 & 302325156 & 5950196787 \\
350000001 & 400000001 & 0.285504 & 365148280 & 7255586684 \\
400000001 & 450000001 & 0.326125 & 441535536 & 8857292252 \\
450000001 & 500000001 & 0.311085 & 479524060 & 9658927478 \\
500000001 & 550000001 & 0.298151 & 543810960 & 11022257029 \\
550000001 & 600000001 & 0.314576 & 591645600 & 12041674931 \\
600000001 & 650000001 & 0.301294 & 639685376 & 13069360680 \\
650000001 & 700000001 & 0.315219 & 660261970 & 13510663499 \\
700000001 & 750000001 & 0.276965 & 728973036 & 14988837355 \\
750000001 & 800000001 & 0.272097 & 772166412 & 15921409781 \\
800000001 & 850000001 & 0.316275 & 838474560 & 17357704112 \\
850000001 & 900000001 & 0.299946 & 855884040 & 17735695879 \\
900000001 & 950000001 & 0.294188 & 921729600 & 19168468472 \\
950000001 & 1000000001 & 0.321118 & 959528080 & 19993096164 \\
\hline
\end{tabular}


When modeling the error term by $x^{1 / 4}$, the local maxima happened to be slowly increasing, which is why we multiplied by an additional $\log x$ obtaining these slowly decreasing local maxima.

Increasing $M$ yields better results, though the improvement is slow to become noticeable.

\begin{tabular}{|r|c||c|r|r|}
\hline \multicolumn{5}{|c|}{ Using the model $\mathscr{M}(x, 4)$} \\
\hline Beginning & End & Max $\leq$ & Where & Sum there \\
\hline 74 & 10001 & 0.520207 & 120 & 602 \\
10001 & 20001 & 0.436010 & $15120-$ & 147800 \\
20001 & 30001 & 0.403803 & 25200 & 259338 \\
30001 & 40001 & 0.377591 & $30240-$ & 316597 \\
40001 & 50001 & 0.399680 & 49140 & 538485 \\
50001 & 60001 & 0.392255 & 50400 & 553570 \\
60001 & 70001 & 0.367556 & 65520 & 736809 \\
70001 & 80001 & 0.359261 & $75240-$ & 856382 \\
80001 & 90001 & 0.353541 & $83160-$ & 954846 \\
90001 & 100000 & 0.397458 & 98280 & 1145047 \\
\hline
\end{tabular}

\begin{tabular}{|r|r||c|r|r|}
\hline \multicolumn{5}{|c|}{ Using the model $\mathscr{M}(x, 4)$} \\
\hline Beginning & \multicolumn{1}{|c|}{ End } & Max $\leq$ & Where & Sum there \\
\hline 74 & 50000001 & 0.520207 & 120 & 602 \\
50000001 & 100000001 & 0.332461 & 82882820 & 1523997698 \\
100000001 & 150000001 & 0.320852 & 134603040 & 2540265823 \\
150000001 & 200000001 & 0.317678 & 165765640 & 3162894841 \\
200000001 & 250000001 & 0.289804 & 232589280 & 4516702124 \\
250000001 & 300000001 & 0.301569 & 274266920 & 5371256127 \\
300000001 & 350000001 & 0.319558 & 319842688 & 6312982612 \\
350000001 & 400000001 & 0.271346 & 365148280 & 7255586684 \\
400000001 & 450000001 & 0.303091 & 419237280 & 8388259211 \\
450000001 & 500000001 & 0.289065 & 465178560 & 9355841003 \\
500000001 & 550000001 & 0.288701 & 522937800 & 10578721101 \\
550000001 & 600000001 & 0.289808 & 583222500 & 11861877982 \\
600000001 & 650000001 & 0.296236 & 639685376 & 13069360680 \\
650000001 & 700000001 & 0.292158 & 678391200 & 13900010069 \\
700000001 & 750000001 & 0.267957 & 730296576 & 15017376156 \\
750000001 & 800000001 & 0.263906 & 772166412 & 15921409781 \\
800000001 & 850000001 & 0.306857 & 838474560 & 17357704112 \\
850000001 & 900000001 & 0.283255 & 868746501 & 18015191334 \\
900000001 & 950000001 & 0.267106 & 913641302 & 18992209828 \\
950000001 & 1000000000 & 0.300615 & 959528080 & 19993096164 \\
\hline
\end{tabular}

See section 14 for a detailed output. Here are the main corollaries, beside Theorem 1.3, that arise from these computations: 
Corollary 6.1. For each $x \in\left[1440,10^{10}\right]$, we have

$$
\Delta(x)=\mathscr{M}(x, 1)+O^{*}\left(0.45 x^{1 / 4} \log x\right)
$$

and we can replace $\mathscr{M}(x, 1)$ by $\mathscr{M}(x, 4)$ in this equality. Moreover, for $x \in\left[2017,10^{10}\right]$,

$$
\Delta(x)=\mathscr{M}(x, 4)+O^{*}\left(0.44 x^{1 / 4} \log x\right) .
$$

Here is the counterpart of Theorem [1.3, when using $\mathscr{M}(x, 1)$ as a model.

Corollary 6.2. For each $x \in\left[4221010,10^{10}\right]$, we have

$$
\Delta(x)=\mathscr{M}(x, 1)+O^{*}\left(0.35 x^{1 / 4} \log x\right) .
$$

Corollary 6.3. For each $x \in\left[3,10^{10}\right]$, we have

$$
\Delta(x)=\mathscr{M}(x, 1)+O^{*}\left(x^{1 / 4} \log x\right) .
$$

Going below $x=3$ does not make much sense: if we extend the range to cover $[2,3]$, the constant 0.9 when $M=4$ becomes 1.7 , but we cannot reach $x=1$, because our upper bound vanishes (since $\log 1=0$ ), but not the difference. A similar remark applies to the case $M=1$.

\section{Numerically COMPARING $\Delta(x)$ TO $\sqrt{x}$}

It is easy to use the bounds of the previous section to compare $\Delta(x)$ with $\sqrt{x}$ when $x$ is somewhat large. The results are then most easily extended to smaller values of $x$ by short computations. We have used the function MajoreDelta with beg $=1$, and $\mathrm{D}=1$ of the following routine:

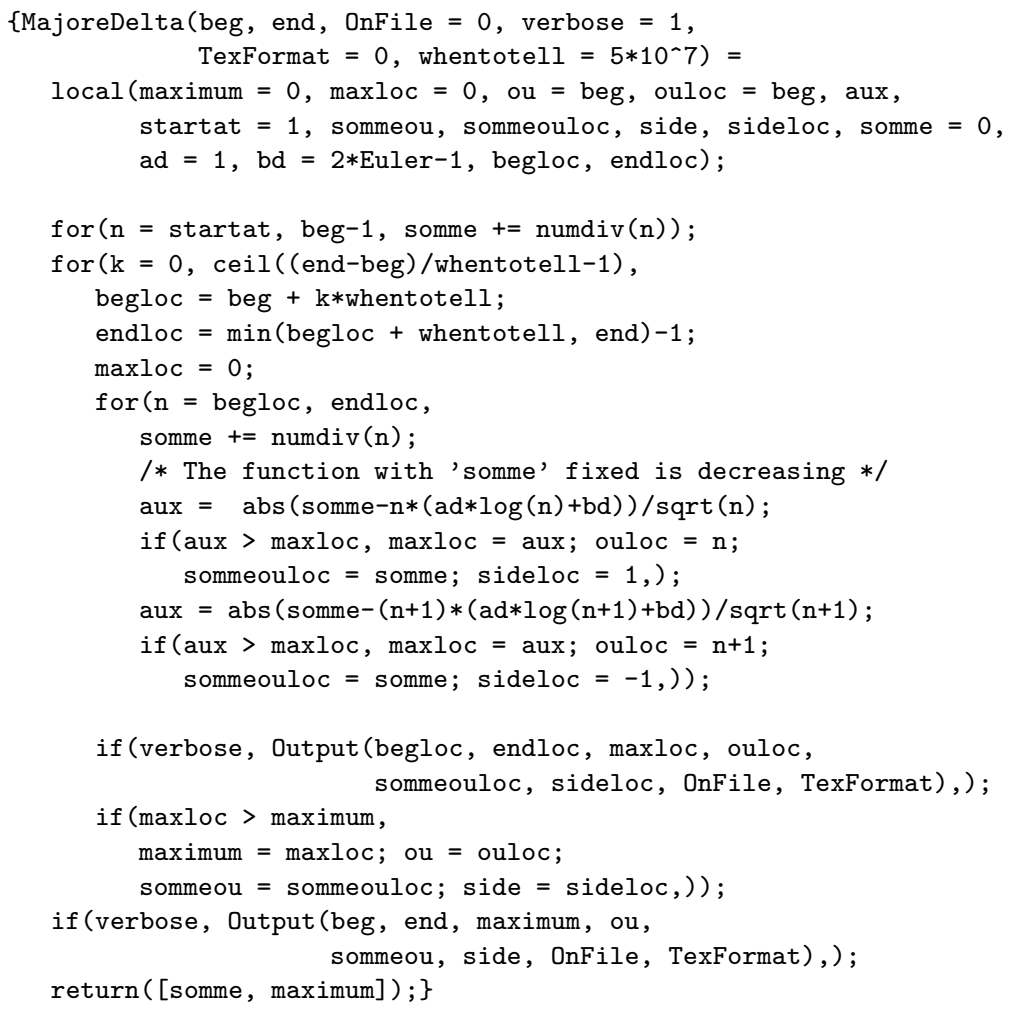


Here is the table obtained, each entry requiring at the beginning about ten minutes and about twenty-five at the end (on a desktop computer).

\begin{tabular}{|r|r||c|r|r|}
\hline Beginning & End & Max $\leq$ & Where & Sum there \\
\hline 1 & 50000001 & 0.960695 & 12 & 35 \\
50000001 & 100000001 & 0.070919 & 82882820 & 1523997698 \\
100000001 & 150000001 & 0.058336 & 135408288 & 2556270358 \\
150000001 & 200000001 & 0.058275 & 165765640 & 3162894841 \\
200000001 & 250000001 & 0.048470 & 219367470 & 4247106335 \\
250000001 & 300000001 & 0.047795 & 253159920 & 4937622542 \\
300000001 & 350000001 & 0.049268 & 302325156 & 5950196787 \\
350000001 & 400000001 & 0.041915 & 353687040 & 7016569614 \\
400000001 & 450000001 & 0.044068 & 403507656 & 8058104197 \\
450000001 & 500000001 & 0.043468 & 479524060 & 9658927478 \\
500000001 & 550000001 & 0.039691 & 529621200 & 10720648283 \\
550000001 & 600000001 & 0.040632 & 562282656 & 11415433396 \\
600000001 & 650000001 & 0.039443 & 639685376 & 13069360680 \\
650000001 & 700000001 & 0.041340 & 660261970 & 13510663499 \\
700000001 & 750000001 & 0.035375 & 728973036 & 14988837355 \\
750000001 & 800000001 & 0.033995 & 768928275 & 15851410875 \\
800000001 & 850000001 & 0.037986 & 838474560 & 17357704112 \\
850000001 & 900000001 & 0.036950 & 855884040 & 17735695879 \\
900000001 & 950000001 & 0.035765 & 921729600 & 19168468472 \\
950000001 & 1000000001 & 0.036828 & 959528080 & 19993096164 \\
\hline
\end{tabular}

Here are some more corollaries:

$$
\left\{\begin{aligned}
& \max _{59} 200<x \leq 10^{10}|\Delta(x)| / \sqrt{x} \leq 0.175, \\
& \max _{7880} 000<x \leq 10^{10}|\Delta(x)| / \sqrt{x} \leq \leq 0.101 \\
& \max _{1.8 \cdot 10^{7}<x \leq 10^{10}}|\Delta(x)| / \sqrt{x} \leq 0.05 .
\end{aligned}\right.
$$

Looking for the bound 0.5 , we find that

Lemma 7.1. When $1981 \leq x \leq 10^{10}$, we have $|\Delta(x)| \leq 0.482 x^{1 / 2}$.

\section{Bounding TWo InTEgrals With $\Delta$}

We consider here, for $\sigma>1$, the integral

$$
I(D, T, \sigma)=\int_{1}^{T} \frac{\Delta(D, u) d u}{u^{\sigma}}
$$

with the aim of bounding $I(D, T, 3 / 2)$ and $I(D, T, 7 / 4)$ explicitly. We abbreviate $I(1, T, \sigma)$ by $I(T, \sigma)$. We define, for $\sigma>1$,

$$
\kappa(D, \sigma)=\sum_{\delta \mid D} \frac{\mu(\delta)}{\delta^{2(\sigma-1)}} \frac{\zeta(\sigma-1)^{2}}{\sigma-1}+\sum_{\delta \mid D} \frac{\mu(\delta)}{\delta^{2}} \frac{-2 \log \delta-\frac{1}{2-\sigma}+2 \gamma-1}{2-\sigma}
$$

and

$$
I_{\sharp}(D, T, \sigma)=\frac{1}{2 i \pi} \int_{c^{\prime}-i \infty}^{c^{\prime}+i \infty} \sum_{\delta \mid D} \frac{\mu(\delta)}{\delta^{2(s+1-\sigma)}} \frac{\zeta^{2}(s) T^{s} d s}{s(s-\sigma+1)}
$$

for $0<c^{\prime}<\sigma-1$. 
Lemma 8.1. We have, when $\sigma \in] 1,2[$,

$$
I(D, T, \sigma)=\kappa(D, \sigma)+\frac{I_{\sharp}(D, T, \sigma)}{T^{\sigma-1}} .
$$

This shows that $I(D, T, \sigma)$ tends to a limit when $T$ goes to infinity (on selecting for instance $\left.c^{\prime}=(\sigma-1) / 2\right)$. Note that $\kappa(1,3 / 2)=0.57413324 \cdots$, which numerically fits, and that $\kappa(1,7 / 4)=0.40765213 \cdots$.

Proof. We start with the case $D=1$. We define

$$
I_{0}(T, \sigma)=\int_{1}^{T} \frac{\sum_{n \leq u} \tau(n) d u}{u^{\sigma}} .
$$

We rewrite this function as follows:

$$
I_{0}(T, \sigma)=\sum_{n \leq T} \tau(n) \int_{n}^{T} \frac{d u}{u^{\sigma}}=\sum_{n \geq 1} \frac{\tau(n)}{n^{\sigma-1}} f_{\sigma}(n / T),
$$

where

$$
f_{\sigma}(v)= \begin{cases}\int_{v}^{1} \frac{d w}{w^{2-\sigma}}=\frac{1-v^{\sigma-1}}{\sigma-1} & \text { when } v \leq 1 \\ 0 & \text { when } v \geq 1\end{cases}
$$

We consider the Mellin transform of $f_{\sigma}$,

$$
\check{f}_{\sigma}(s)=\int_{0}^{\infty} f_{\sigma}(v) v^{s-1} d v=\frac{1}{s(s+\sigma-1)},
$$

which is readily computed so that

$$
f_{\sigma}(v)=\frac{1}{2 i \pi} \int_{2-i \infty}^{2+i \infty} \check{f}_{\sigma}(s) v^{-s} d s .
$$

This gives us

$$
\begin{aligned}
I_{0}(T, \sigma) & =\frac{1}{2 i \pi} \int_{2-i \infty}^{2+i \infty} \sum_{n \geq 1} \frac{\tau(n)}{n^{s+\sigma-1}} \check{f}_{\sigma}(s) T^{s} d s \\
& =\frac{1}{2 i \pi} \int_{2-i \infty}^{2+i \infty} \zeta^{2}(s+\sigma-1) \frac{T^{s} d s}{s(s+\sigma-1)} .
\end{aligned}
$$

The poles of the integrand are in $2-\sigma$ (a double pole), in 0 (a simple pole) and in $1-\sigma$ (a simple pole). Note that, in the vicinity of $s=2-\sigma$, we have

$$
\zeta^{2}(s+\sigma-1)=\frac{1}{(s+\sigma-2)^{2}}+\frac{2 \gamma}{s+\sigma-2}+O(1)
$$

and that

$$
\frac{T^{s}}{s(s+\sigma-1)}=\frac{T^{2-\sigma}}{2-\sigma}\left(1+(s+\sigma-2)\left(\log T-\frac{1}{2-\sigma}-1\right)\right)+O\left((s+\sigma-2)^{2}\right)
$$

so that

$$
\frac{\zeta^{2}(s+\sigma-1) T^{s}}{s(s+\sigma-1)}=\frac{T^{2-\sigma}}{2-\sigma}\left(\frac{1}{(s+\sigma-2)^{2}}+\frac{1}{s+\sigma-2}\left(\log T-\frac{1}{2-\sigma}-1+2 \gamma\right)\right)+O(1) .
$$


The Cauchy Residue Theorem yields:

$$
\begin{aligned}
I_{0}(T, \sigma)=\frac{T^{2-\sigma}}{2-\sigma}\left(\log T-\frac{1}{2-\sigma}-1+\right. & 2 \gamma)+\frac{\zeta(\sigma-1)^{2}}{\sigma-1} \\
& +\frac{1}{2 i \pi} \int_{c-i \infty}^{c+i \infty} \zeta^{2}(s+\sigma-1) \frac{T^{s} d s}{s(s+\sigma-1)}
\end{aligned}
$$

for any $1-\sigma<c<0$. We need the condition $c>1-\sigma$ to ensure the convergence of the integral. Indeed, we know that

$$
\left|\zeta^{2}(a+i b)\right| \ll(|b|+2)^{-(1-a)} \log ^{2}(|b|+2)
$$

when $0 \leq a \leq 1$. Better bounds are known, but the size of $\left|\zeta^{2}(a+i b)\right|$ can indeed be as large as $|b|$, and this implies that we can ensure the convergence of the integral only when $c>1-\sigma$.

Let us remark here that

$$
\int_{1}^{T} \frac{u(\log u+2 \gamma-1)}{u^{\sigma}} d u=T^{2-\sigma} \frac{\log T+(\sigma-2)^{-1}+2 \gamma-1}{2-\sigma}-\frac{(\sigma-2)^{-1}+2 \gamma-1}{2-\sigma} .
$$

The lemma follows readily when $D=1$. For a general $D$, we appeal to (4.5), and deduce that

$$
I(D, T, \sigma)=\sum_{\delta \mid D} \frac{\mu(\delta)}{\delta^{2(\sigma-1)}}\left(I\left(T / \delta^{2}, \sigma\right)-\int_{1 / \delta^{2}}^{1} \frac{\log u+2 \gamma-1}{u^{\sigma-1}} d u\right) .
$$

We notice that

$$
\int_{1 / \delta^{2}}^{1} \frac{\log u+2 \gamma-1}{u^{\sigma-1}} d u=\frac{(\sigma-2)^{-1}+2 \gamma-1}{2-\sigma}-\frac{(\sigma-2)^{-1}+2 \gamma-1-2 \log \delta}{(2-\sigma) \delta^{2(2-\sigma)}}
$$

We need to bound $I_{\sharp}(T, 1 / 2)$ and $I_{\sharp}(T, 3 / 4)$ explicitly.

Lemma 8.2. We have

$$
I_{\sharp}(T, 3 / 2)=I_{\sharp}(T, 7 / 4)+\frac{1}{2}+O^{*}\left(\frac{9}{2} / T^{0.22}\right) .
$$

Proof. Let us first compute the derivative of $I_{\sharp}(T, \sigma)$ with respect to $\sigma$. We readily find that

$$
\begin{aligned}
I_{\sharp}^{\prime}(T, \sigma) & =\frac{-1}{2 i \pi} \int_{c^{\prime}-i \infty}^{c^{\prime}+i \infty} \frac{\zeta^{2}(s) T^{s} d s}{s(s-\sigma+1)^{2}} \\
& =\frac{-1}{4(\sigma-1)^{2}}-\frac{1}{2 i \pi} \int_{-\frac{1}{4}-i \infty}^{-\frac{1}{4}+i \infty} \frac{\zeta^{2}(s) T^{s} d s}{s(s-\sigma+1)^{2}} .
\end{aligned}
$$

At this level, we employ the functional equation of the Riemann zeta function in the form

$$
\zeta(s)=2^{s} \pi^{s-1} \sin (\pi s / 2) \Gamma(1-s) \zeta(1-s)
$$

to get, when $\sigma \in[3 / 2,7 / 4]$, and with $c^{\prime}=-\delta>-1 / 4$,

$$
\begin{aligned}
\mid \frac{1}{2 i \pi} & \int_{-\delta-i \infty}^{-\delta+i \infty} \frac{\zeta^{2}(s) T^{s} d s}{s(s-\sigma+1)^{2}} . \mid \\
& \leq \frac{\zeta(1+\delta)^{2}}{T^{\delta} \pi^{3+2 \delta} 2^{2 \delta}} \int_{0}^{\infty} \frac{|\sin (\pi(-\delta+i y) / 2) \Gamma(1-\delta+i y)|^{2} d y}{\left|\delta+i y \|\left(\delta-\frac{1}{4}\right)+i y\right|^{2}} .
\end{aligned}
$$


On selecting $\delta=0.22$, we compute that

$$
I_{\sharp}^{\prime}(T, \sigma)=\frac{-1}{4(\sigma-1)^{2}}+O^{*}\left(18 / T^{0.22}\right) .
$$

First, we use GP to produce the following bounds.

Lemma 8.3. We have

$$
\max _{1 \leq T \leq 100000} T^{1 / 4}|I(T, 7 / 4)-\kappa(7 / 4)| \leq 0.302
$$

and also

$$
\max _{1260 \leq T \leq 10000000} T^{1 / 4}|I(T, 7 / 4)-\kappa(7 / 4)| \leq 0.00979
$$

Proof. This is obtained by using the function MajoreResteJ.

Let us now evaluate $I(T, 7 / 4)$ by using Lemma 3.1

Lemma 8.4. We have $\max _{T \geq 1} T^{1 / 4}|\kappa(7 / 4)-I(T, 7 / 4)| \leq 4.000001$.

Proof. We find that, on using (3.1) and noticing that $I(\infty, 7 / 4)=\kappa(7 / 4)$,

$$
|\kappa(7 / 4)-I(T, 7 / 4)| \leq \int_{T}^{\infty} \frac{d u}{u^{5 / 4}}+\frac{1}{2} \int_{T}^{\infty} \frac{d u}{u^{7 / 4}} \leq \frac{4+\frac{2}{3} T^{-1 / 2}}{T^{1 / 4}} .
$$

Lemma 8.3 takes care of the small values of $T$.

Once Lemma 9.3 has been established, we will have access to the following improvement:

Lemma 8.5. We have $\max _{T \geq 1} T^{1 / 4}|\kappa(7 / 4)-I(T, 7 / 4)| \leq 1.90$.

See Lemma 11.1 for a further improvement.

Lemma 8.6. We have $\max _{T \geq 1}|I(T, 7 / 4)| \leq 0.479$.

The computations we ran lead us to think that $I(T, 3 / 4) \leq \kappa(3 / 4)$ is plausible. We formulate the following general question:

Question 8.7. Is it true that, for $\sigma \in[3 / 2,7 / 4]$, we have

$$
\forall T \geq 1, \quad I(T, \sigma) \leq \kappa(\sigma) ?
$$

This question is surprising as some positivity mechanism seems hidden. A proof (or disproof) assuming GRH would also be welcome. The range $[3 / 2,7 / 4]$ may be extended, but $\sigma=2$ seems to have a special status. The reader will understand the conjecture stated in the introduction by noticing that $I(\infty, \sigma)=\kappa(\sigma)$. We mention here the papers [18, (2.2)], 9] and [5] where the Dirichlet series $\int_{1}^{\infty} \Delta(u) d u / u^{s}$ is studied.

Proof. A numerical computation using the GP calculator and the function MajoreJ below shows that

$$
\max _{1 \leq T \leq 10^{7}}|I(T, 7 / 4)| \leq 0.4077
$$

and, on using Lemma 8.4, the lemma follows readily.

Lemma 8.8. We have $\max _{T \geq 1}|I(T, 3 / 2)| \leq 4.71$. 
This bound is fairly poor since we believe that $|I(T, 3 / 2)| \leq \kappa(3 / 2)=0.574 \cdots$. Once Lemma 9.3 will be established, we will have access to the following improvement:

Lemma 8.9. We have $\max _{T \geq 1}|I(T, 3 / 2)| \leq 2.61$.

Proof. We have, by Lemma 8.1 and 8.2 .

$$
\begin{aligned}
I(T, 3 / 2) & =\kappa(3 / 2)+\frac{I_{\sharp}(T, 3 / 2)}{T^{1 / 2}} \\
& =\kappa(3 / 2)+\frac{1}{2 T^{1 / 2}}+\frac{I_{\sharp}(T, 7 / 4)}{T^{1 / 2}}+O^{*}\left(\frac{9}{2} / T^{0.22}\right) \\
& =\kappa(3 / 2)+\frac{1}{2 T^{1 / 2}}+T^{1 / 4}(I(T, 7 / 4)-\kappa(7 / 4))+O^{*}\left(\frac{9}{2} / T^{0.22}\right) .
\end{aligned}
$$

We appeal to Lemma 8.4 or to Lemma 8.5 to bound the third summand. A numerical computation using the GP calculator shows that

$$
\max _{1 \leq T \leq 10^{7}}|I(T, 3 / 2)| \leq \kappa(3 / 2) .
$$

\section{A FIRST BOUND}

We use Corollary 3.2 with $D=1$ to get

$$
\begin{aligned}
|G(x, T)| \leq \frac{7}{4}+\left(7+\left(x T^{-3}\right)^{1 / 4}+\left(x T^{-3}\right)^{1 / 2}\right) \frac{\sqrt{T}+\frac{1}{2}}{4} & \\
& +\left(\frac{\sqrt{x}}{8 T}+\frac{\sqrt{T}}{12}\right) \int_{1}^{T} \frac{\Delta(u) d u}{u^{3 / 2}}+\frac{9 x^{1 / 4}}{16} \int_{1}^{T} \frac{\Delta(u) d u}{u^{7 / 4}} .
\end{aligned}
$$

We appeal to Lemma 8.6 and 8.8 to get

$$
\begin{aligned}
|G(x, T)| \leq \frac{7}{4}+\left(7+\left(x T^{-3}\right)^{1 / 4}+\left(x T^{-3}\right)^{1 / 2}\right) & \frac{\sqrt{T}+\frac{1}{2}}{4} \\
& +4.71\left(\frac{\sqrt{x}}{8 T}+\frac{\sqrt{T}}{12}\right)+0.479 \frac{9 x^{1 / 4}}{16} .
\end{aligned}
$$

We select

$$
T=\left(\frac{\sqrt{357}}{6}+\frac{3}{2}\right)^{-4 / 3} x^{1 / 3}=c x^{1 / 3}
$$

and get

$$
\begin{aligned}
|G(x, T)| \leq \frac{7}{4}+\left(7+c^{-3 / 4}+c^{-3 / 2}\right) \frac{\sqrt{c} x^{1 / 6}+\frac{1}{2}}{4} & \\
& +4.71\left(\frac{x^{1 / 6}}{8 c}+\frac{\sqrt{c} x^{1 / 6}}{12}\right)+0.479 \frac{9 x^{1 / 4}}{16},
\end{aligned}
$$

i.e.,

$$
|G(x, T)| \leq 0.27 x^{1 / 4}+7.7 x^{1 / 6}+6.0 .
$$

The global bound we obtain is

$$
|\Delta(x)| \leq 1.146 x^{1 / 3} \log x-10.5 x^{1 / 3}+8.93 x^{1 / 4}+11.4 x^{1 / 6}+5.91 .
$$

When we divide by $x^{1 / 3} \log x$, the function first decreases and then increases up to 1.146. It is $\leq 1.146$ when $x \geq 379$. 
Lemma 9.1. We have

$$
\max _{14 \leq x \leq 10^{6}} \frac{|\Delta(x)|}{x^{1 / 3} \log x}=\frac{|\Delta(36)|}{36^{1 / 3} \log 36}=\frac{140}{36^{1 / 3} \log 36} \leq 0.4593 .
$$

Lemma 9.2. When $x \geq 3$, we have $|\Delta(x)| \leq 1.146 x^{1 / 3} \log x$.

See also Lemma 10.7. As a consequence we get:

Lemma 9.3. When $x \geq 121$, we have $|\Delta(x)| \leq 0.76 x^{1 / 2}$.

See also Lemma 10.6. As a further consequence we get:

Lemma 9.4. When $x \geq 4033$, we have $|\Delta(x)| \leq 0.475 x^{1 / 2}$.

We have $|\Delta(x)|>0.48 x^{1 / 2}$ when $x=4032$.

\section{TAKing adVAntage of $D$}

We can now use Lemma 8.9 and also use the parameter $D$. A direct computation gives us the following bounds.

Lemma 10.1. We have $\max _{1 \leq x \leq 10^{7}}|\Delta(2, x)| \leq 0.883 x^{1 / 2}$.

We have $\max _{1 \leq x \leq 10^{7}}|\Delta(6, x)| \leq 0.927 x^{1 / 2}$.

Lemma 10.2. We have $\max _{x \geq 1}|\Delta(2, x)| \leq 0.883 x^{1 / 2}$.

We have $\max _{x \geq 1}|\Delta(6, x)| \leq 0.950 x^{1 / 2}$.

Proof. We use (4.5) together with Lemma 9.4 when available, as well as Lemma10.1 for the smaller values.

Lemma 10.3. We have

$$
\begin{aligned}
& \max _{1 \leq T \leq 10^{7}}|I(2, T, 7 / 4)| \leq 0.902, \\
& \max _{1 \leq T \leq 10^{7}}|I(6, T, 7 / 4)| \leq 0.0945, \\
& \max _{1 \leq T \leq 10^{7}}|I(6, T, 3 / 2)| \leq 0.131 .
\end{aligned}
$$

We also have $I\left(6,10^{7}, 3 / 2\right)=-0.056667+O^{*}\left(10^{-6}\right)$.

Proof. We use the PARI/GP package.

Lemma 10.4. We have, for all $T \geq 1,|I(2, T, 7 / 4)| \leq 0.953$.

We have, for all $T \geq 1,|I(6, T, 7 / 4)| \leq 0.163$.

Proof. We use, when $T \geq T_{0}=10^{7}$ and, on using Lemma 9.4.

$$
\begin{aligned}
|I(D, T, 7 / 4)| & \leq\left|I\left(D, T_{0}, 7 / 4\right)\right|+\int_{T_{0}}^{T} \sum_{\delta \mid D} 0.475 \frac{d u}{\delta u^{5 / 4}} \\
& \leq\left|I\left(D, T_{0}, 7 / 4\right)\right|+1.90 \frac{\sigma(D)}{D T_{0}^{1 / 4}} .
\end{aligned}
$$

A numerical application using Lemma 10.3 concludes the proof.

Lemma 10.5. We have, for all $T \geq 1,|I(2, T, 3 / 2)| \leq 3.91$.

We have, for all $T \geq 1,|I(6, T, 3 / 2)| \leq 5.98$. 
Proof. We reuse (8.8), together with (8.9), to write

$$
\begin{aligned}
I(D, T, \sigma)=\sum_{\delta \mid D} \frac{\mu(\delta) I\left(T / \delta^{2}, \sigma\right)}{\delta^{2(\sigma-1)}}-\sum_{\delta \mid D} & \frac{\mu(\delta)}{\delta^{2(\sigma-1)}} \frac{(\sigma-2)^{-1}+2 \gamma-1}{2-\sigma} \\
& +\sum_{\delta \mid D} \frac{\mu(\delta)}{\delta^{2}} \frac{(\sigma-2)^{-1}+2 \gamma-1-2 \log \delta}{2-\sigma} .
\end{aligned}
$$

On using (4.4), we get

$$
\begin{aligned}
& I(D, T, \sigma)=\sum_{\delta \mid D} \frac{\mu(\delta) I\left(T / \delta^{2}, \sigma\right)}{\delta^{2(\sigma-1)}}-\sum_{\delta \mid D} \frac{\mu(\delta)}{\delta^{2(\sigma-1)}} \frac{(\sigma-2)^{-1}+2 \gamma-1}{2-\sigma} \\
&+B(D)-\frac{A(D)}{(2-\sigma)^{2}} .
\end{aligned}
$$

This leads to

$$
I(D, T, 3 / 2)=\sum_{\delta \mid D} \frac{\mu(\delta) I\left(T / \delta^{2}, 3 / 2\right)}{\delta}-2(2 \gamma-3) \frac{\phi(D)}{D}+B(D)-4 A(D) .
$$

By appealing to Lemma 8.2, we get:

$$
\begin{aligned}
I(D, T, 3 / 2) & =\kappa(D, 3 / 2) \frac{\phi(D)}{D}+\sum_{\delta \mid D} \frac{\mu(\delta) I_{\#}\left(T / \delta^{2}, 3 / 2\right)}{\sqrt{T} \delta} \\
& -2(2 \gamma-3) \frac{\phi(D)}{D}+B(D)-4 A(D) \\
& =\frac{\phi(D)}{2 D \sqrt{T}}+O^{*}\left(\sum_{\delta \mid D} \frac{1}{\delta^{0.56} T^{0.72}}\right)+\sum_{\delta \mid D} \frac{\mu(\delta) I_{\#}\left(T / \delta^{2}, 7 / 4\right)}{\sqrt{T} \delta} \\
& +\kappa(D, 3 / 2) \frac{\phi(D)}{D}-2(2 \gamma-3) \frac{\phi(D)}{D}+B(D)-4 A(D) \\
& =O^{*}\left(\sum_{\delta \mid D} \frac{1}{\delta^{0.56} T^{0.72}}\right)+\sum_{\delta \mid D} \frac{\mu(\delta) T^{1 / 4}\left(I\left(T / \delta^{2}, 7 / 4\right)-\kappa(7 / 4)\right)}{\delta} \\
& +\kappa(D, 3 / 2) \frac{\phi(D)}{D}+\frac{\phi(D)}{2 D \sqrt{T}}-2(2 \gamma-3) \frac{\phi(D)}{D}+B(D)-4 A(D) .
\end{aligned}
$$

Lemma 8.5 applies.

Next, we use a direct computation with $T$ and $c$ from (9.1) and get, with $D=6$ :

$$
|\Delta(x)| \leq 0.764 x^{1 / 3} \log x-4.505 x^{1 / 3}+4.755 x^{1 / 4}+10.30 x^{1 / 6}+7 / 4 .
$$

As a consequence we get:

Lemma 10.6. When $x \geq 421$, we have $|\Delta(x)| \leq 0.688 x^{1 / 2}$.

Proof. Use the above inequality (10.2) when $x \geq 10^{9}$, Lemma 7.1 when $x \geq 1981$ and MajoreDelta otherwise.

Lemma 10.7. When $x \geq 9995$, we have $|\Delta(x)| \leq 0.764 x^{1 / 3} \log x$.

Proof. The right-hand side of inequality (10.2) divided by $x^{1 / 3} \log x$ is decreasing and then increasing.

The third bound of Theorem 1.1 is a further consequence of this bound. 


\section{SECOND ROUND}

We can try to use our better estimates to improve on the final result. The next lemma indeed improves on Lemma 8.5 but the global improvement is of no consequence.

Lemma 11.1. We have $\max _{T \geq 1} T^{1 / 4}|\kappa(7 / 4)-I(T, 7 / 4)| \leq 1.83$.

Proof. For $T \leq 10^{7}=T_{0}$, this follows from Lemma 8.3. For larger $T$ 's, we use (10.2) to show that $|\kappa(D, 7 / 4)-I(D, T, 7 / 4)|$ is not more than

$$
\int_{T}^{\infty}\left(0.764 u^{1 / 3} \log u-4.505 u^{1 / 3}+4.755 u^{1 / 4}+10.30 u^{1 / 6}+7 / 4\right) \frac{d u}{u^{7 / 4}}
$$

i.e., $T^{1 / 4}|\kappa(7 / 4)-I(T, 7 / 4)|$ is not more than

$$
0.764 \frac{\frac{12}{5} \log T+\left(\frac{12}{5}\right)^{2}}{T^{1 / 6}}-4.505 \frac{\frac{12}{5}}{T^{1 / 6}}+4.755 \frac{2}{T^{1 / 4}}+10.30 \frac{\frac{12}{7}}{T^{1 / 3}}+\frac{7}{4} \frac{\frac{3}{4}}{T^{1 / 2}} .
$$

This function is decreasing, and takes a value $\leq 1.83$ at $T=10^{7}$.

We thus get $\max _{T \geq 1}|I(2, T, 3 / 2)| \leq 3.79$ and $\max _{T \geq 1}|I(2, T, 3 / 2)| \leq 5.79$.

We use MajDelta with $T$ and $c$ from (9.1) and get, with $D=6$ :

$$
|\Delta(x)| \leq 0.764 x^{1 / 3} \log x-4.505 x^{1 / 3}+4.755 x^{1 / 4}+10.11 x^{1 / 6}+7 / 4,
$$

which is a very modest improvement.

\section{Proof of Corollary 2.1}

Since $\zeta_{K}(s) \leq \zeta(s)^{n}$ for every $s>1$ and every number field of degree $n$ (see [14, Chapter 7 , Corollary 3]), we find that (since $n=2$ here)

$$
h_{K} \leq \sum_{m \leqslant b_{K} \sqrt{d_{K}}} \tau(m) .
$$

On invoking Theorem 1.1 we get

$$
h_{K} \leqslant \sqrt{\frac{d_{K}}{12}} \log d_{K}+\sqrt{\frac{d_{K}}{3}}\left(2 \gamma-1-\log \sqrt{3}+0.961\left(\frac{d_{K}}{3}\right)^{-1 / 4}\right),
$$

and it is easily seen that

$$
2 \gamma-1-\log \sqrt{3}+0.961\left(d_{K} / 3\right)^{-1 / 4}<0
$$

as soon as $d_{K} \geqslant 108$.

\section{Proof of Corollary 2.2}

An integration by parts yields

$$
\begin{aligned}
\sum_{n \leq t} \frac{\tau(n)}{n} & =\sum_{n \leq t} \tau(n)\left(\int_{n}^{t} \frac{d t}{t}+\frac{1}{t}\right) \\
& =\int_{1}^{t}(u \log u+(2 \gamma-1) u+\Delta(u)) \frac{d u}{u^{2}}+\log t+2 \gamma-1+\frac{\Delta(t)}{t} \\
& =\frac{1}{2} \log ^{2} t+A \log t+B+\frac{\Delta(t)}{t}-\int_{t}^{\infty} \frac{\Delta(u) d u}{u^{2}}
\end{aligned}
$$


for constants $A=2 \gamma$ and $B=\gamma^{2}-\gamma_{1}$. By Theorem 1.1, we find that

$$
R(t)=t^{1 / 3}\left|\frac{\Delta(t)}{t}-\int_{t}^{\infty} \frac{\Delta(u) d u}{u^{2}}\right| \leq 3 \cdot 0.961 / t^{1 / 6}
$$

which is not more than 1.16 provided $t$ is larger than 236 . We readily write a routine to complete the proof. Below are some partial results.

\begin{tabular}{|c||c|}
\hline Interval & $R(t) \leq$ \\
\hline$[0,1]$ & 1.16 \\
{$[1,2]$} & 0.60 \\
{$[2,3]$} & 0.57 \\
{$[3,4]$} & 0.72 \\
{$[4,5]$} & 0.48 \\
\hline
\end{tabular}

\begin{tabular}{|c||c|}
\hline Interval & $R(t) \leq$ \\
\hline$[5,6]$ & 0.48 \\
{$[6,7]$} & 0.74 \\
{$[7,8]$} & 0.43 \\
{$[8,9]$} & 0.61 \\
{$[9,10]$} & 0.52 \\
\hline
\end{tabular}

\section{TABLES}

We give the values obtained at some points, so that future authors can check their and our results. We can also start computations anew from one of these points. These computations have taken about ten days on a decent computer.

\begin{tabular}{|r|c||c|c|r|}
\hline \multicolumn{6}{|c|}{ Using the model $\mathscr{M}(x, 4)$} \\
\hline Beginning & End & Max $\leq$ & Where & Sum there \\
\hline 1000000000 & 1050000000 & 0.274960 & 1033783300 & 21617363398 \\
1050000000 & 1100000000 & 0.300485 & 1061260200 & 22219769642 \\
1100000000 & 1150000000 & 0.289880 & 1124565312 & 23610355396 \\
1150000000 & 1200000000 & 0.309673 & 1183291200 & 24903544168 \\
1200000000 & 1250000000 & 0.281165 & 1209300625 & 25477231529 \\
1250000000 & 1300000000 & 0.259583 & 1286477760 & 27182768219 \\
1300000000 & 1350000000 & 0.278165 & 1349790904 & 28585396325 \\
1350000000 & 1400000000 & 0.287948 & 1357738256 & 28761673191 \\
1400000000 & 1450000000 & 0.271429 & 1449339220 & 30796727408 \\
1450000000 & 1500000000 & 0.260179 & 1493821875 & 31787089049 \\
1500000000 & 1550000000 & 0.283459 & 1536464160 & 32737721129 \\
1550000000 & 1600000000 & 0.270070 & 1591890300 & 33975109938 \\
1600000000 & 1650000000 & 0.285854 & 1619982000 & 34602998536 \\
1650000000 & 1700000000 & 0.292418 & 1678295250 & 35907926633 \\
1700000000 & 1750000000 & 0.281376 & 1732250520 & 37117138632 \\
1750000000 & 1800000000 & 0.288213 & 1774936800 & 38074990519 \\
1800000000 & 1850000000 & 0.269459 & 1814760150 & 38969526424 \\
1850000000 & 1900000000 & 0.259731 & 1853948320 & 39850647721 \\
1900000000 & 1950000000 & 0.277342 & 1919056152 & 41316379639 \\
1950000000 & 2000000000 & 0.243022 & 1980250000 & 42696013532 \\
\hline
\end{tabular}




\begin{tabular}{|r|c||c|c|r|}
\hline \multicolumn{5}{|c|}{ Using the model $\mathscr{M}(x, 4)$} \\
\hline Beginning & End & Max $\leq$ & Where & Sum there \\
\hline 2000000000 & 2050000000 & 0.293896 & 2035173616 & 43935895580 \\
2050000000 & 2100000000 & 0.276613 & 2067566622 & 44667854438 \\
2100000000 & 2150000000 & 0.251389 & 2122520400 & 45910757214 \\
2150000000 & 2200000000 & 0.252292 & 2190178000 & 47442935997 \\
2200000000 & 2250000000 & 0.280737 & 2242590948 & 48631324066 \\
2250000000 & 2300000000 & 0.248571 & 2272574080 & 49311700641 \\
2300000000 & 2350000000 & 0.268572 & 2325892808 & 50522582467 \\
2350000000 & 2400000000 & 0.279156 & 2366582400 & 51447477213 \\
2400000000 & 2450000000 & 0.256179 & 2401245000 & 52235927480 \\
2450000000 & 2500000000 & 0.270924 & 2458573065 & 53541031206 \\
2500000000 & 2550000000 & 0.264865 & 2545875360 & 55531071836 \\
2550000000 & 2600000000 & 0.269957 & 2559702020 & 55846525595 \\
2600000000 & 2650000000 & 0.249882 & 2618708448 & 57193584643 \\
2650000000 & 2700000000 & 0.270260 & 2670564018 & 58378495847 \\
2700000000 & 2750000000 & 0.300742 & 2731307040 & 59767766081 \\
2750000000 & 2800000000 & 0.275779 & 2750075328 & 60197295267 \\
2800000000 & 2850000000 & 0.246828 & 2814240537 & 61666736191 \\
2850000000 & 2900000000 & 0.263185 & 2851560000 & 62522060994 \\
2900000000 & 2950000000 & 0.261988 & 2934660966 & 64428396764 \\
2950000000 & 3000000000 & 0.283013 & 2987643784 & 65645054999 \\
\hline
\end{tabular}

\begin{tabular}{|r|c||c|c|r|}
\hline \multicolumn{5}{|c|}{ Using the model $\mathscr{M}(x, 4)$} \\
\hline Beginning & End & Max $\leq$ & Where & Sum there \\
\hline 3000000000 & 3050000000 & 0.273352 & 3023790600 & 66475644081 \\
3050000000 & 3100000000 & 0.296701 & 3072928352 & 67605433767 \\
3100000000 & 3150000000 & 0.244745 & 3130246086 & 68924291232 \\
3150000000 & 3200000000 & 0.279620 & 3183780600 & 70157047562 \\
3200000000 & 3250000000 & 0.261684 & 3239964000 & 71451767900 \\
3250000000 & 3300000000 & 0.259188 & 3277140048 & 72309009478 \\
3300000000 & 3350000000 & 0.246630 & 3339610560 & 73750462665 \\
3350000000 & 3400000000 & 0.265684 & 3367538928 & 74395265220 \\
3400000000 & 3450000000 & 0.260423 & 3413610945 & 75459468392 \\
3450000000 & 3500000000 & 0.263876 & 3480115590 & 76996733092 \\
3500000000 & 3550000000 & 0.279600 & 3549873600 & 78610565016 \\
3550000000 & 3600000000 & 0.285749 & 3576846340 & 79234940728 \\
3600000000 & 3650000000 & 0.263195 & 3622600800 & 80294547476 \\
3650000000 & 3700000000 & 0.260356 & 3650296881 & 80936229741 \\
3700000000 & 3750000000 & 0.282580 & 3726736650 & 82708325799 \\
3750000000 & 3800000000 & 0.247660 & 3786588436 & 84096959797 \\
3800000000 & 3850000000 & 0.260401 & 3839553025 & 85326592376 \\
3850000000 & 3900000000 & 0.236244 & 3883096910 & 86338060202 \\
3900000000 & 3950000000 & 0.250083 & 3904000500 & 86823797338 \\
3950000000 & 4000000000 & 0.246883 & 3987985851 & 88776488468 \\
\hline
\end{tabular}


It is not apparent here, but the maxima have all been attained at the beginning of the intervals $[N, N+1)$, for the program would otherwise have attached a minus sign at the back of the data "Where".

\begin{tabular}{|r|c||c|c|r|}
\hline \multicolumn{5}{|c|}{ Using the model $\mathscr{M}(x, 4)$} \\
\hline Beginning & End & Max $\leq$ & Where & Sum there \\
\hline 4000000000 & 4050000000 & 0.245688 & 4025648718 & 89652741254 \\
4050000000 & 4100000000 & 0.290455 & 4096960560 & 91312823163 \\
4100000000 & 4150000000 & 0.257367 & 4116441888 & 91766549369 \\
4150000000 & 4200000000 & 0.248970 & 4176455300 & 93164858229 \\
4200000000 & 4250000000 & 0.251872 & 4214402192 & 94049464392 \\
4250000000 & 4300000000 & 0.248332 & 4289204400 & 95794228028 \\
4300000000 & 4350000000 & 0.252001 & 4334643000 & 96854722988 \\
4350000000 & 4400000000 & 0.242011 & 4372030080 & 97727660193 \\
4400000000 & 4450000000 & 0.263651 & 4434229920 & 99180648960 \\
4450000000 & 4500000000 & 0.258938 & 4485181896 & 100371538069 \\
4500000000 & 4550000000 & 0.249314 & 4500699138 & 100734334151 \\
4550000000 & 4600000000 & 0.257573 & 4599891522 & 103054728842 \\
4600000000 & 4650000000 & 0.268573 & 4635160200 & 103880282398 \\
4650000000 & 4700000000 & 0.280269 & 4651785616 & 104269535886 \\
4700000000 & 4750000000 & 0.271884 & 4747743000 & 106517355799 \\
4750000000 & 4800000000 & 0.253353 & 4797640320 & 107686979253 \\
4800000000 & 4850000000 & 0.272364 & 4843238478 & 108756281301 \\
4850000000 & 4900000000 & 0.265096 & 4864923000 & 109264946520 \\
4900000000 & 4950000000 & 0.238144 & 4917146130 & 110490367074 \\
4950000000 & 5000000000 & 0.248641 & 4973705100 & 111818154382 \\
\hline
\end{tabular}

\begin{tabular}{|r|c||c|c|r|}
\hline \multicolumn{5}{|c|}{ Using the model $\mathscr{M}(x, 4)$} \\
\hline Beginning & End & Max $\leq$ & Where & Sum there \\
\hline 5000000000 & 5050000000 & 0.286181 & 5027022945 & 113070441623 \\
5050000000 & 5100000000 & 0.244395 & 5091750720 & 114591475202 \\
5100000000 & 5150000000 & 0.258298 & 5119404040 & 115241550386 \\
5150000000 & 5200000000 & 0.261626 & 5176785636 & 116590954111 \\
5200000000 & 5250000000 & 0.251771 & 5240781400 & 118096648865 \\
5250000000 & 5300000000 & 0.247590 & 5262850320 & 118616068971 \\
5300000000 & 5350000000 & 0.253317 & 5308652478 & 119694375880 \\
5350000000 & 5400000000 & 0.277900 & 5379593492 & 121365298487 \\
5400000000 & 5450000000 & 0.245229 & 5449523400 & 123013321106 \\
5450000000 & 5500000000 & 0.264486 & 5462614192 & 123321929177 \\
5500000000 & 5550000000 & 0.281960 & 5500150656 & 124207003555 \\
5550000000 & 5600000000 & 0.241436 & 5560748820 & 125636390340 \\
5600000000 & 5650000000 & 0.253000 & 5615407644 & 126926247584 \\
5650000000 & 5700000000 & 0.246390 & 5668548484 & 128180792385 \\
5700000000 & 5750000000 & 0.261162 & 5746455792 & 130020921855 \\
5750000000 & 5800000000 & 0.261145 & 5779524000 & 130802295723 \\
5800000000 & 5850000000 & 0.235602 & 5849192160 & 132449113120 \\
5850000000 & 5900000000 & 0.258207 & 5869321932 & 132925096267 \\
5900000000 & 5950000000 & 0.243802 & 5929741468 & 134354172920 \\
5950000000 & 6000000000 & 0.247427 & 5975287568 & 135431862589 \\
\hline
\end{tabular}




\begin{tabular}{|r|c||c|c|r|}
\hline \multicolumn{5}{|c|}{ Using the model $\mathscr{M}(x, 4)$} \\
\hline Beginning & End & Max $\leq$ & Where & Sum there \\
\hline 6000000000 & 6050000000 & 0.257078 & 6047581276 & 137143152604 \\
6050000000 & 6100000000 & 0.264247 & 6076125240 & 137819065525 \\
6100000000 & 6150000000 & 0.237060 & 6145856660 & 139470848065 \\
6150000000 & 6200000000 & 0.254862 & 6183777600 & 140369443674 \\
6200000000 & 6250000000 & 0.250717 & 6240605010 & 141716492287 \\
6250000000 & 6300000000 & 0.258793 & 6269789344 & 142408485236 \\
6300000000 & 6350000000 & 0.236728 & 6337831710 & 144022371582 \\
6350000000 & 6400000000 & 0.257214 & 6367561200 & 144727750832 \\
6400000000 & 6450000000 & 0.234042 & 6430236132 & 146215266132 \\
6450000000 & 6500000000 & 0.243262 & 6456122508 & 146829827370 \\
6500000000 & 6550000000 & 0.229275 & 6529368096 & 148569291100 \\
6550000000 & 6600000000 & 0.262629 & 6588000720 & 149962313570 \\
6600000000 & 6650000000 & 0.256246 & 6627458574 & 150900066265 \\
6650000000 & 6700000000 & 0.247609 & 6686825190 & 152311411807 \\
6700000000 & 6750000000 & 0.276045 & 6727772700 & 153285180309 \\
6750000000 & 6800000000 & 0.236609 & 6760131840 & 154054885524 \\
6800000000 & 6850000000 & 0.256445 & 6822102224 & 155529366196 \\
6850000000 & 6900000000 & 0.251456 & 6862382760 & 156488075487 \\
6900000000 & 6950000000 & 0.245043 & 6914587680 & 157730946631 \\
6950000000 & 7000000000 & 0.267887 & 6960231180 & 158817929605 \\
\hline
\end{tabular}

\begin{tabular}{|r|c||c|c|r|}
\hline \multicolumn{5}{|c|}{ Using the model $\mathscr{M}(x, 4)$} \\
\hline Beginning & End & Max $\leq$ & Where & Sum there \\
\hline 7000000000 & 7050000000 & 0.238635 & 7045913952 & 160859238973 \\
7050000000 & 7100000000 & 0.261163 & 7095895040 & 162050472517 \\
7100000000 & 7150000000 & 0.260647 & 7123107862 & 162699202748 \\
7150000000 & 7200000000 & 0.245588 & 7153692680 & 163428442105 \\
7200000000 & 7250000000 & 0.272719 & 7245201600 & 165611085040 \\
7250000000 & 7300000000 & 0.253628 & 7289919000 & 166678092011 \\
7300000000 & 7350000000 & 0.261667 & 7329609000 & 167625369333 \\
7350000000 & 7400000000 & 0.246222 & 7351690752 & 168152485810 \\
7400000000 & 7450000000 & 0.235580 & 7436388960 & 170174940560 \\
7450000000 & 7500000000 & 0.258627 & 7453473300 & 170583004109 \\
7500000000 & 7550000000 & 0.236566 & 7549916010 & 172887291892 \\
7550000000 & 7600000000 & 0.236878 & 7559867700 & 173125136377 \\
7600000000 & 7650000000 & 0.235268 & 7611602866 & 174361811910 \\
7650000000 & 7700000000 & 0.267827 & 7679106060 & 175975934558 \\
7700000000 & 7750000000 & 0.250518 & 7742196000 & 177485064361 \\
7750000000 & 7800000000 & 0.237627 & 7794947646 & 178747294449 \\
7800000000 & 7850000000 & 0.240256 & 7808001006 & 179059687402 \\
7850000000 & 7900000000 & 0.241885 & 7870262400 & 180550027446 \\
7900000000 & 7950000000 & 0.240581 & 7905966138 & 181404884029 \\
7950000000 & 8000000000 & 0.276060 & 7961011704 & 182723158806 \\
\hline
\end{tabular}




\begin{tabular}{|r|c||c|c|r|}
\hline \multicolumn{5}{|c|}{ Using the model $\mathscr{M}(x, 4)$} \\
\hline Beginning & End & Max $\leq$ & Where & Sum there \\
\hline 8000000000 & 8050000000 & 0.250616 & 8003807296 & 183748324280 \\
8050000000 & 8100000000 & 0.237212 & 8055421920 & 184985053658 \\
8100000000 & 8150000000 & 0.224514 & 8126722674 & 186694022338 \\
8150000000 & 8200000000 & 0.267527 & 8193921120 & 188305238294 \\
8200000000 & 8250000000 & 0.249990 & 8222771718 & 188997157791 \\
8250000000 & 8300000000 & 0.239580 & 8264446302 & 189996811511 \\
8300000000 & 8350000000 & 0.236183 & 8308550250 & 191054967561 \\
8350000000 & 8400000000 & 0.254539 & 8375178258 & 192653972096 \\
8400000000 & 8450000000 & 0.250009 & 8403113964 & 193324558500 \\
8450000000 & 8500000000 & 0.253240 & 8458325316 & 194650159683 \\
8500000000 & 8550000000 & 0.236732 & 8547846636 & 196800293764 \\
8550000000 & 8600000000 & 0.231778 & 8586658080 & 197732763494 \\
8600000000 & 8650000000 & 0.247982 & 8613789264 & 198384712006 \\
8650000000 & 8700000000 & 0.251100 & 8669286000 & 199718536031 \\
8700000000 & 8750000000 & 0.244156 & 8747676300 & 201603194893 \\
8750000000 & 8800000000 & 0.247581 & 8766483264 & 202055456509 \\
8800000000 & 8850000000 & 0.248287 & 8825690880 & 203479517971 \\
8850000000 & 8900000000 & 0.234675 & 8882685504 & 204850727934 \\
8900000000 & 8950000000 & 0.248082 & 8944167540 & 206330308775 \\
8950000000 & 9000000000 & 0.243354 & 8951421360 & 206504901825 \\
\hline
\end{tabular}

\begin{tabular}{|r|r||c|c|r|}
\hline \multicolumn{5}{|c|}{ Using the model $\mathscr{M}(x, 4)$} \\
\hline Beginning & End & Max $\leq$ & Where & Sum there \\
\hline 9000000000 & 9050000000 & 0.275712 & 9001398276 & 207707961207 \\
9050000000 & 9100000000 & 0.238592 & 9072415200 & 209417978179 \\
9100000000 & 9150000000 & 0.289532 & 9137256975 & 210979790121 \\
9150000000 & 9200000000 & 0.242404 & 9169786080 & 211763475672 \\
9200000000 & 9250000000 & 0.235055 & 9229445316 & 213201075792 \\
9250000000 & 9300000000 & 0.225647 & 9269774283 & 214173095441 \\
9300000000 & 9350000000 & 0.246018 & 9303571200 & 214987813705 \\
9350000000 & 9400000000 & 0.235136 & 9385928200 & 216973647295 \\
9400000000 & 9450000000 & 0.239579 & 9432100650 & 218087297681 \\
9450000000 & 9500000000 & 0.265125 & 9495486000 & 219616479788 \\
9500000000 & 9550000000 & 0.229020 & 9532008024 & 220497772162 \\
9550000000 & 9600000000 & 0.246084 & 9562200508 & 221226435660 \\
9600000000 & 9650000000 & 0.241961 & 9614588560 & 222490991420 \\
9650000000 & 9700000000 & 0.272435 & 9686476956 & 224226715659 \\
9700000000 & 9750000000 & 0.240663 & 9712890915 & 224864607349 \\
9750000000 & 9800000000 & 0.240285 & 9789225600 & 226708477160 \\
9800000000 & 9850000000 & 0.232302 & 9834292260 & 227797345066 \\
9850000000 & 9900000000 & 0.222418 & 9880665810 & 228918004951 \\
9900000000 & 9950000000 & 0.223151 & 9924314400 & 229973012094 \\
9950000000 & 10000000000 & 0.259598 & 9976913352 & 231244609722 \\
\hline
\end{tabular}




\section{ACKNOWLEDGEMENT}

Thanks are due to the referee for his/her very careful reading of the first version of this paper.

\section{REFERENCES}

[1] O. Bordellès. Explicit upper bounds for the average order of $d_{n}(m)$ and application to class number. JIPAM. J. Inequal. Pure Appl. Math., 3(3):Article 38, 15 pp. (electronic), 2002. MR.1917797 (2003e:11118)

[2] Y. Cheng and S.W. Graham. Explicit estimates for the Riemann zeta function. Rocky Mountain J. Math., 34(4):1261-1280, 2004. MR2095256 (2005f:11179)

[3] M.W. Coffey. New results on the Stieltjes constants: asymptotic and exact evaluation. J. Math. Anal. Appl., 317(2):603-612, 2006. MR2209581 (2007g:11106)

[4] J.-M Deshouillers and F. Dress. Sommes de diviseurs et structure multiplicative des entiers. Acta Arith., 49(4):341-375, 1988. MR937932 (89e:11054)

[5] J. Furuya, Y. Tanigawa, and W. Zhai. Dirichlet series obtained from the error term in the Dirichlet divisor problem. Monatsh. Math., 160(4):385-402, 2010. MR2661321

[6] A. Granville and O. Ramaré. Explicit bounds on exponential sums and the scarcity of squarefree binomial coefficients. Mathematika, 43(1):73-107, 1996. MR.1401709 (97m:11023)

[7] M.N. Huxley and A. Ivić. Subconvexity for the Riemann zeta-function and the divisor problem. Bull. Cl. Sci. Math. Nat. Sci. Math., (32):13-32, 2007. MR2386169

[8] A. Ivić. The Riemann zeta-function. The theory of the Riemann zeta-function with applications. A Wiley-Interscience Publication. New York, John Wiley \& Sons. XVI, 517 pp., 1985. MR.792089 (87d:11062)

[9] A. Ivić. On the integral of the error term in the Dirichlet divisor problem. Bull. Cl. Sci. Math. Nat. Sci. Math., (25):29-45, 2000. MR.1842813 (2002e:11127)

[10] R. Kreminski. Newton-Cotes integration for approximating Stieltjes (generalized Euler) constants. Math. Comp., 72(243):1379-1397 (electronic), 2003. MR1972742 (2004a:11140)

[11] M. Le. Upper bounds for class numbers of real quadratic fields. Acta Arith., 68:141-145, 1994. MR.1305196 (95j:11101)

[12] Ž. Linkovski1. The lower and upper bound estimates of the mean values of numerical functions. Rev. Roumaine Math. Pures Appl., 15:69-73, 1970. MR0262195 (41:6805)

[13] T. Meurman. On the mean square of the Riemann zeta-function. Quart. J. Math. Oxford Ser. (2), 38(151):337-343, 1987. MR907241 (88j:11054)

[14] W. Narkiewicz. Elementary and analytic theory of algebraic numbers. Springer Monographs in Mathematics. Springer-Verlag, Berlin, third edition, 2004. MR.2078267 (2005c:11131)

[15] O. Ramaré. On Snirel'man's constant. Ann. Scu. Norm. Pisa, 21:645-706, 1995. http:// math.univ-lille1.fr/ ramare/Maths/Article.pdf. MR.1375315(97a:11167)

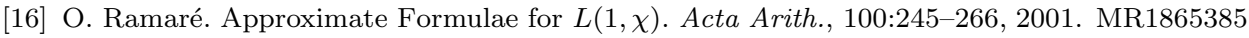
(2002k:11144)

[17] O. Ramaré and R. Rumely. Primes in arithmetic progressions. Math. Comp., 65:397-425, 1996. MR1320898 (97a:11144)

[18] R. Sitaramachandra Rao. An integral involving the remainder term in the Piltz divisor problem. Acta Arith., 48(1):89-92, 1987. MR893465 (88h:11068)

[19] H. Riesel and R.C. Vaughan. On sums of primes. Arkiv för mathematik, 21:45-74, 1983. MR706639 (84m:10042)

[20] PARI/GP, version 2.4.3. Bordeaux, 2008. http://pari.math.u-bordeaux.fr/.

[21] G. Voronoï. Sur un problème de calculs des fonctions asymptotiques. J. Reine Angew. Math., 126:241-282, 1903

[22] G. Voronoï. Sur une fonction transcendante et ses applications à la sommation de quelques séries. Ann. Sci. École Norm. Sup. (3), 21:207-267, 1904. MR1509041

[23] G. Voronoï. Sur une fonction transcendante et ses applications à la sommation de quelques séries (suite). Ann. Sci. École Norm. Sup. (3), 21:459-533, 1904. MR1509047 
Département de Mathématiques, Université de Blida, 270 route de soumaa, 09000 BlidA, AlgÉrie

E-mail address: djameberkan@gmail.fr

2, allée de la combe, 43000 Aiguilhe, France

E-mail address: borde43@wanadoo.fr

CNRS / Laboratoire Paul Painlevé, Université Lille 1, 59655 Villeneuve D'AscQ CEDEx, France

E-mail address: ramare@math.univ-lille1.fr 